\title{
HOMOTOPY SECTIONS OF ONE DIMENSIONAL IDEALS OVER A LAURENT POLYNOMIAL RING
}

\author{
Ratnesh Kumar Mishra \\ AIAS, Department of Mathematics \\ Amity University Uttar Pradesh \\ Noida, INDIA
}

\begin{abstract}
Let $R$ be a commutative Noetherian ring and $I$ be a one dimensional ideal of the Laurent polynomial ring $R\left[X, X^{-1}\right]$ that contains a doubly monic polynomial. Define $I(1):=<\{f(1): f \in I\}>$. Let $I / I^{2}$ be genereted by $n \geq 2$ elements over $R\left[X, X^{-1}\right] / I$. Then any set of $n$ generators of $I(1)$ over $R$ may not be lifted to a set of $n$ generators of $I$ over $R\left[X, X^{-1}\right]$ by describing an example of one dimensional ideal.
\end{abstract}

AMS Subject Classification: 13E05, 13E15, 13C10

Key Words: projective modules, free modules, Laurent polynomial ring, Noetherian ring and number of generators

\section{Introduction}

Let $R$ be a commutative Noetherian ring of finite Krull dimension with identity and $I$ be an ideal of the Laurent polynomial ring $R\left[X, X^{-1}\right]$. Let $M$ be a finitely generated $R$-module. We denote $\mu(M)$ to be the least number of elements required to generate $M$ as an $R$-module. The comparison of the minimal numbers of generators of $I$ and of its module $I / I^{2}$, denoted by $\mu(I)$ and $\mu\left(I / I^{2}\right)$, has been an enliven area of interest (see [1], [5], [6], [11], [14], [15] and [17]). The co-normal bundle $I / I^{2}$ of an ideal $I$ in a ring $R\left[X, X^{-1}\right]$ is an $R\left[X, X^{-1}\right] / I$ module. Many algebraic properties of this module are intertwined with those

$\begin{array}{lr}\text { Received: } & \text { December } 6,2016 \\ \text { Revised: } & \text { June 1, } 2017 \\ \text { Published: } & \text { August 3, 2017 }\end{array}$

(c) 2017 Academic Publications, Ltd. url: www.acadpubl.eu 
of the ideal $I$. Define $I(1)=<\{f(1): f \in I\}>$. In general, $\mu(I(1)) \leq \mu(I)$ and $\mu\left(I / I^{2}\right) \leq \mu(I) \leq \mu\left(I / I^{2}\right)+1$.

Any set of $n$ generators of the maximal ideal $\left(X_{1}, X_{2}, \ldots, X_{n-1}\right)$ of $K\left[X_{1}, X_{2}\right.$, $\left.\ldots, X_{n-1}\right]$ can be lifted (via the map setting $X_{n}$ to 0 ) to a set of $n$ generators of the maximal ideal $\left(X_{1}, X_{2}, \ldots, X_{n}\right)$ of $K\left[X_{1}, X_{2}, \ldots\right.$,

$\left.X_{n}\right]$. For $n=2$, let $\left(F_{1}\left(X_{1}\right), F_{2}\left(X_{1}\right)\right)$ generate the ideal $\left(X_{1}\right)$ of $K\left[X_{1}\right]$. We wish to lift these to a set of two generators of maximal ideals $\left(X_{1}, X_{2}\right)$ of $K\left[X_{1}, X_{2}\right]$. It is easy to see that we can perform Euclidean transformations on $F_{1}, F_{2}$ and using the Euclidean transformations we may assume that $F_{1}=X_{1}$ and $F_{2}=0$. Then for these generators, the lift $\left(X_{1}, X_{2}\right)$ works.

Since lifting of generators is a natural problem which have been studied by many mathematicians in various fashion (see [4], [7], [12], [10], [18]). Here we are giving some examples on lifting problem. Let $X$ be an integral, projective variety of co-dimension two, degree $d$ and dimension $r$. Suppose $Y$ is its general hyperplane section. The problem of lifting generators of minimal degree $\sigma$ from the homogeneous ideal of $X$ is studied in ([12]). Answers in terms of relations between $d$ and $\sigma$ are known for $r=1,2$. For $r=1$, the Laudal's "Generalized trisecant lemma" is given in ([2], [9]). For $r=2$, there is the analogous result in ([13]). In ([18]), let $X$ be an integral, projective variety of dimension $n$ and degree $d$ in $P^{N}$, defined over an algebraically closed field $K$ of characteristic zero. Consider the hyperplane section $Y=X \bigcap K$ of $X$, where $K \cong P^{N-1}$ is a general hyperplane in $P^{N}$. The "Lifting problem" is the problem of finding conditions on $d, N, n$ and $s$ such that any degree $s$ hypersurface in $P^{n-1}$ containing $Y$ can be lifted to a hypersurface in $P^{N}$ containing $X$. Motivated by this problem, in [10] Mandal proved that the following:

Problem 1.1. [10] If $R$ is a commutative Noetherian ring, $I$ is an ideal of Laurent polynomial ring $R\left[X, X^{-1}\right]$ containing a doubly monic polynomial, and $I / I^{2}$ is generated by $n$ elements over $R\left[X, X^{-1}\right] / I$, where

$$
n \geq \operatorname{dim}\left(R\left[X, X^{-1}\right] / I\right)+2,
$$

then $I$ is generated by $n$ elements over $R\left[X, X^{-1}\right]$. Note that $I(1)$ is also generated by $n$ elements, where $I(1)$ is an ideal of $R$ by putting $X=1$.

In $([4])$, lifting problem in various cases has been discussed. If $I$ is an ideal of $R[X]$, then $I(0):=<\{f(0): f \in I\}>$ is also an ideal of $R$ so it is very natural to ask whether a set of generators for $I(0)$ can be lifted to a set of generators for $I$. One interesting consequence of this is in the Laurent case. We consider the problem for one dimensional ideals of Laurent polynomial rings. A natural question is: can we lift any set of $n$ generators of $I(1)$ to a set of 
$n$ generators of $I$ ? The objective of the paper is to come out with a negative answer of the above problem in case of the one dimensional ideals, i.e., a set of $\mathrm{n}$ generators of $I(1)$ may not be lifted to a set of $n$ generators of $I$.

\section{Preliminaries}

In this section, we define some terms used in this paper and provide certain standard results without proof. We hope that this will improve the readability and understanding of proof of the paper.

An ideal $I$ of a ring $R$ is called zero dimensional if every prime ideal of $R$ containing $I$ is a maximal ideal, that is, $\operatorname{dim}(R / I)=0$. For example every maximal ideal is a zero dimensional ideal. Further if $I$ is the intersection of finitely many maximal ideals, then $\operatorname{dim}(R / I)=0$ and the converse is also true if $I$ is a reduced ideal. An ideal $I$ of a $\operatorname{ring} R$ is called one dimensional if $\operatorname{dim}(R / I)=1$. A polynomial $f$ in Laurent polynomial $\operatorname{ring} R\left[X, X^{-1}\right]$ is said to be doubly monic polynomial if coefficient of the highest degree term and the lowest degree term are units. We say that an ideal $I$ is efficiently generated if $\mu(I)=\mu\left(I / I^{2}\right)$. We have a theorem of Mandal [10] about efficient generation of ideals.

Theorem 2.1. [10] Let $I$ be an ideal of $R\left[X, X^{-1}\right]$ over a commutative Noetherian ring $R$. Suppose that $I$ contains a doubly monic polynomial and $\mu\left(I / I^{2}\right) \geq \operatorname{dim}\left(R\left[X, X^{-1}\right] / I\right)+2$. Then ideal $I$ is efficiently generated.

The following theorem proved by Kumar and Mandal in [7] shows the positive result in the case of zero dimensional ideal.

Theorem 2.2. [7] Let $R$ be a commutative Noetherian ring and $I$ be an ideal of the Laurent polynomial ring $R\left[X, X^{-1}\right]$ that contains a doubly monic polynomial. Suppose $P$ is a projective $R$ - module of rank

$$
n \geq \operatorname{dim}\left(R\left[X, X^{-1}\right] / I\right)+2 .
$$

Let $S: P \rightarrow I(1)$ and $\phi: R\left[X, X^{-1}\right] \rightarrow I / I^{2}$ be two surjective homomorphisms such that $\phi(1) \equiv S \bmod \left(X-1, I(1)^{2}\right)$. Then there exists a surjective homomorphism $\psi: R\left[X, X^{-1}\right] \rightarrow I$ such that $\psi(1)=S$ and $\psi$ lifts $\phi$.

\section{Main Result}

Now we give an example to show that a set of $\mathrm{n}$ generators of $I(1)$ may not be lifted to a set of $n$ generators of $I$ in the case of one dimensional ideals. 
Example 3.1. Let $R=\mathbb{Q}[U, V, W]$, where $\mathbb{Q}$ is the field of rational numbers and $I=\left(U, V, X+1+\phi_{p}(W)\right)$ be an ideal of $R\left[X, X^{-1}\right]$ generated by $\left(U, V, X+1+\phi_{p}(W)\right)$, where $\phi_{p}(W)$ is a cyclotomic polynomial in $W$ of degree $p-1$ and $\phi_{p}(W)$ is irreducible over $\mathbb{Q}$ and $X+1+\phi_{p}(W) \in \mathbb{Q}[W]\left[X, X^{-1}\right]$ has a root in $\mathbb{Q}[W]$. When we set $X=1$ the ring acquires a unit $W$ which can not be lifted to original ring. We have $I(1)=\left(U, V, \phi_{p}(W)\right)$. Note that $R\left[X, X^{-1}\right] / I=$ $\mathbb{Q}\left[U, V, W, X, X^{-1}\right] /\left(U, V, X+1+\phi_{p}(W)\right) \cong \mathbb{Q}\left[W, X, X^{-1}\right] /\left(X+1+\phi_{p}(W)\right)$ and $R / I(1)=\mathbb{Q}[U, V, W] /\left(U, V, \phi_{p}(W)\right) \cong \mathbb{Q}[W] / \phi_{p}(W)$ is a field, because $\phi_{p}(W)$ is irreducible polynomial over $\mathbb{Q}$. Clearly,

$$
\operatorname{dim}\left(R\left[X, X^{-1}\right] / I\right)=\operatorname{dim}\left(\mathbb{Q}\left[W, X, X^{-1}\right] /\left(X+1+\phi_{p}(W)\right)\right)=1
$$

and $\operatorname{dim}(R / I(1))=0, \mu(I)=h t(I)=3$ and $\mu(I(1))=h t(I(1))=3$. This implies that $I$ and $I(1)$ are complete intersection ideals of $R\left[X, X^{-1}\right]$ and $R$ respectively. Hence $I / I^{2}$ and $I(1) / I(1)^{2}$ are free modules of rank 3 over $R\left[X, X^{-1}\right] / I$ and $R / I(1)$ respectively. It follows that $\left\{U+I(1)^{2}, V+I(1)^{2}, \phi_{p}(W)+I(1)^{2}\right\}$ is a free basis of $I(1) / I(1)^{2}$ over $R / I(1)$. Since $\left\{F_{1}+I(1)^{2}, F_{2}+I(1)^{2}, F_{3}+I(1)^{2}\right\}$ is also a free basis of $I(1) / I(1)^{2}$ over $R / I(1)$, where each $F_{i}=f_{i}(U, V, W)$ for $i=1,2,3$. So there exists an invertible matrix $T_{1} \in G L_{3}(R / I(1))$ such that

$$
\left[\begin{array}{c}
F_{1}+I(1)^{2} \\
F_{2}+I(1)^{2} \\
F_{3}+I(1)^{2}
\end{array}\right]=T_{1}\left[\begin{array}{c}
U+I(1)^{2} \\
V+I(1)^{2} \\
\phi_{p}(W)+I(1)^{2}
\end{array}\right]
$$

where $\left\{F_{1}, F_{2}, F_{3}\right\}$ generates $I(1)$ and $T_{1}=\left[a_{i j}(U, V, W)+I(1)\right], 1 \leq i, j \leq 3$. Suppose that $\left\{F_{1}, F_{2}, F_{3}\right\}$ has a lifting $\left\{G_{1}, G_{2}, G_{3}\right\}$ to a generating set of $I$, where each $g_{i}(U, V, W, X+1)=G_{i}$ and each $g_{i}(U, V, W, 0)=F_{i}$, for $i=1,2,3$. It follows that $\left\{G_{1}+I^{2}, G_{2}+I^{2}, G_{3}+I^{2}\right\}$ is a free basis of $I / I^{2}$ over $R\left[X, X^{-1}\right] / I$. Since $\left\{U+I^{2}, V+I^{2}, X+1+\phi_{p}(W)+I^{2}\right\}$ is also a free basis of $I / I^{2}$ over $R\left[X, X^{-1}\right] / I$, there exists an invertible matrix $T_{2} \in G L_{3}\left(R\left[X, X^{-1}\right] / I\right)$ such that

$$
\left[\begin{array}{c}
G_{1}+I^{2} \\
G_{2}+I^{2} \\
G_{3}+I^{2}
\end{array}\right]=T_{2}\left[\begin{array}{c}
U+I^{2} \\
V+I^{2} \\
X+1+\phi_{p}(W)+I^{2}
\end{array}\right]
$$

Suppose $T_{2}=\left[b_{i j}(U, V, W, X+1)+I\right]$, where $b_{i j}(U, V, W, X+1) \in R\left[X, X^{-1}\right], 1 \leq$ $i, j \leq 3$. Let $f(W)$ be a root of $X+1+\phi_{p}(W)$ in $\mathbb{Q}[W]$. Since $R\left[X, X^{-1}\right] / I \cong$ $\mathbb{Q}\left[W, X, X^{-1}\right] /\left(X+1+\phi_{p}(W)\right), \operatorname{Det}\left[b_{i j}(0,0, W\right.$,

$f(W)]$ is free from $W$ and is a non-zero member of $\mathbb{Q}$. Substituting $X=1$, we get

$$
\left[\begin{array}{c}
F_{1}+I(1)^{2} \\
F_{2}+I(1)^{2} \\
F_{3}+I(1)^{2}
\end{array}\right]=\left[b_{i j}(U, V, W, 0)+I(1)\right]\left[\begin{array}{c}
U+I(1)^{2} \\
V+I(1)^{2} \\
\phi_{p}(W)+I(1)^{2}
\end{array}\right],
$$


Let $\alpha$ be a root of $\phi_{p}(W)$. Then this implies that

$$
\operatorname{Det}\left[a_{i j}(0,0, \alpha)\right]=\operatorname{Det}\left[b_{i j}(0,0, f(0))\right],
$$

which is a member of $\mathbb{Q}$. Thus for an example, if we take

$$
T_{1}=\left[\begin{array}{ccc}
W+I(1) & 0+I(1) & 0+I(1) \\
0+I(1) & 1+I(1) & 0+I(1) \\
0+I(1) & 0+I(1) & 1+I(1)
\end{array}\right],
$$

then

$$
\operatorname{Det}\left[\begin{array}{lll}
\alpha & 0 & 0 \\
0 & 1 & 0 \\
0 & 0 & 1
\end{array}\right]=\alpha,
$$

which is not a member of $\mathbb{Q}$, for $\phi_{p}(W)$ is irreducible over $\mathbb{Q}$ of degree greater than 1. Therefore,

$$
\left[\begin{array}{l}
F_{1}+I(1)^{2} \\
F_{2}+I(1)^{2} \\
F_{3}+I(1)^{2}
\end{array}\right]=\left[\begin{array}{ccc}
W+I(1) & 0+I(1) & 0+I(1) \\
0+I(1) & 1+I(1) & 0+I(1) \\
0+I(1) & 0+I(1) & 1+I(1)
\end{array}\right]\left[\begin{array}{c}
U+I(1)^{2} \\
V+I(1)^{2} \\
\phi_{p}(W)+I(1)^{2}
\end{array}\right]^{.}
$$

Hence, $\left\{F_{1}, F_{2}, F_{3}\right\}$ does not have a lifting to a generating set of $I$, that is, the generating set $\left\{U, V, \phi_{p}(W)\right\}$ of $I(1)$ can not be lifted to a generating set of $I$.

\section{Acknowledgment}

Author sincerely thank Prof. Shiv Datt Kumar, M.N.N.I.T. Allahabad and Prof. Raja Sridharan, TIFR, Mumbai for stimulated discussions for improvements of the manuscript.

\section{References}

[1] R. Gilmer, B. Nashier and W. Nicholas, Generators of ideals containing monics, Proceeding of Arch. Math. (Basel), 49, No. 5, (1987), 407-413.

[2] L. Gruson, Ch. Peskine, Section plane D'une courbe gauche: Postulation, enumerative geometry, Prog. in Math, Birkhäuser, 24, No. 3, (1982), 33-35.

[3] S. K. Gupta and M. P. Murthy, Suslin work on linear groups over polynomial rings and serre conjecture, Indian Statistical Institute, New Delhi, (1979), 405-434. 
[4] S. D. Kumar, Some Problems in commutative algebra, D, Phil. Thesis, University of Allahabad, Allahabad, 2002.

[5] S. D. Kumar and R. K. Mishra Lifting of generators of ideals to Laurent polynomial ring, Beitrge zur Algebra und Geometrie / Contributions to Algebra and Geometry, 54, No. 1, 2013, 147-153, doi: 10.1007/s13366-012-0102-x.

[6] S. D. Kumar and P. Singh, On a variant of Bertini's theorem and generators of ideals of a polynomial ring with monic polynomials, Int. J. of Algebra, 3, No. 17,(2009), 863-872. (http://www.m-hikari.com/ija/ija-password-2009/ija-password1720-2009/kumarIJA17-20-2009.pdf).

[7] S. D. Kumar and S. Mandal, Some results on generators of ideals, J. Pure and Applied Algebra, 169, No. 1, (2002), 29-32, doi: 10.1016/S0022-4049(01)00061-5.

[8] R. G. López, On the number of generators of modules over Laurent polynomial rings, Manuscripta Mathematica, Springer-Verlag, 76, (1992), 45-57.

[9] O. A. Laudel, A generalized trisecant lemma, Lecture Notes in Mathematics, SpringerVerlag, Berlin, Heidelberg, and Newyork, 687 (1978), 112-149.

[10] S. Mandal, Number of generators for modules over laurent polynomial rings, J. Alg, 80, No. 2, (1983), 306-313.

[11] S. Mandal, On efficient generation of ideals, Invent. Math., 75 (1984), 59-67.

[12] E. Mezzetti, Differential geometric methods for the lifting problem and linear systems on plane curves, arxiv. alg.-geom/ 9309005v1, 46, No. 23 September, 1993.

[13] E. Mezzetti, I. Raspanti, A Loudal-type theorem for surfaces in $P^{4}$, Rend. Sem. Mat. Univ. Pal. Torino,(to appear).

[14] R. K. Mishra, S. Awasthi and S. D. Kumar, A lifting problem on zero dimensional ideals over laurent polynomial ring, Journal of International Academy of Physical Sciences, 16, No. 1, (2012), 69-74.

[15] N. Mohan Kumar, On two conjectures about polynomial rings, Invent. Math., 46, No. $3,(1978), 225-236$.

[16] S. Mandal, Homotopy sections of projective modules, J. Alg. Geo., 1 (1992), 636-646.

[17] B. Nashier, Monic polynomial and generating ideal efficiently, Proceeding of American Mathematical Society, 95, No. 3, (1985), 338-340, doi: 10.1090/S0002-9939-19850806066-0.

[18] M. Roggero, Lifting problem in co-dimension 2 and initial ideals, Bulletin of the Iranian Mathematical Society, 30, No. 2, (2004), 39-66. 